\title{
The effect of vortex generators on a jet in a cross-flow
}

\author{
K. B. M. Q. Zaman ${ }^{\text {a) }}$ and J. K. Foss ${ }^{\text {b) }}$ \\ NASA Lewis Research Center, Cleveland, Ohio 44135
}

(Received 3 April 1996; accepted 20 August 1996)

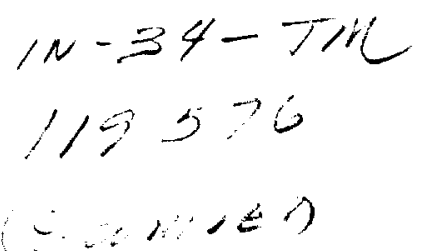

The effect of vortex generators in the form of tabs on the penetration and spreading of a jet in a cross-flow has been studied experimentally. It is found that the tab has very little effect when placed on the leeward side, i.e., on the downstream edge of the jet nozzle relative to the free-stream flow. A study of the static pressure distribution reveals significantly lower pressures on the leeward side. Thus, when placed on that side the tab does not produce a "pressure hill" of sufficient magnitude that is the primary source of streamwise vorticity in the flow field over the tab. This qualitatively explains the ineffectiveness. In comparison, there is a significant effect on the flow field when the tab is placed on the windward side. The sense of vorticity generated by the tab in the latter configuration is opposite to that of the bound vortex pair that otherwise characterizes the flow. Thus, the strength of the bound vortex pair is diminished and the jet penetration is reduced. (c) 1997 American Institute of Physics. [S1070-6631(97)01601-2]

\section{INTRODUCTION}

The effect of vortex generators in the form of tabs on free jets has been investigated in several previous studies (Ahuja and Brown, ${ }^{\prime}$ Zaman et al. ${ }^{2}$ ). The tabs produce streamwise vortex pairs, which, with a suitable combination, can be quite effective in increasing the mixing and spreading of free jets. The increase in jet spreading produced by the tabs has been shown to exceed that achieved by other methods, e.g., through the use of asymmetric nozzles or artificial excitation. ${ }^{3}$ The tabs are also just as effective at supersonic conditions as they are at subsonic conditions. These results prompted an investigation to determine if the tabs would also promote mixing for a jet in a cross-flow (Liscinsky et al. ${ }^{4}$ ), a configuration that is essential in combustors as well as many other engineering applications.

Based on past studies, (e.g., Keffer and Baines, ${ }^{5}$ Kamotani and Greber; ${ }^{6}$ Fearn and Weston; $;^{7}$ Moussa et al. $;^{8}$ and Fric and Roshko ${ }^{9}$ ), it is well known that a main feature of a jet in a cross-flow is the formation and persistence of a pair of counter-rotating vortices, sometimes referred to as the "bound vortex pair." There are other characteristic vortex systems in the flow that may depend on the jet/cross-flow velocity ratio in a complex manner. ${ }^{9-12}$ The bound vortex pair, however, is a dominant feature, which, detectable even in the time-averaged flow field at all velocity ratios, persists hundreds of diameters from the nozzle (Pratte and Baines ${ }^{13}$ ). A cursory study of the sense of rotation of the bound vortex pair and that of a vortex pair generated by a tab suggests that the tab would be most effective when placed, relative to the cross-stream flow, on the downstream edge of the nozzle. Then the vortex pair generated by the tab would be expected to reinforce the bound vortex pair. This was tried in the work of Ref. 4. Unfortunately, the results were not encouraging. The penetration of the jet into the cross-flow as well as the mixing were affected little, if at all, by the tabs.

The results of Ref. 4 contrasted the dramatic effect of

a) Aerospace Engineer.

b) National Research Council Associate. tabs, with comparable geometry and size, on free jets. ${ }^{1-3,14}$ The reason for the ineffectiveness had remained unclear. This provided the motivation for the present investigation. A set of experiments were planned with the objectives of independently verifying the ineffectiveness of the tab for a jet in a cross-flow, carrying out detailed measurements on the distributions of vorticity and other flow field properties, and attempting to provide an explanation.

\section{EXPERIMENTAL FACILITY AND PROCEDURE}

The experiments were conducted in a low-speed wind tunnel with a $76 \mathrm{~cm} \times 51 \mathrm{~cm}$ test section. The open circuit, induction-type, tunnel had a 16:1 contraction section at the inlet, which, together with five screens, yielded a flow with less than $0.1 \%$ turbulence intensity. As shown in Fig. 1, a $2.23 \mathrm{~cm}$ diam $(D)$ jet discharged normally from the floor of the test section. The nozzle exit was located $0.45 \mathrm{~m}$ from the end of the tunnel contraction section. The approach boundary layer was inferred to be laminar, with an estimated momentum thickness of about $0.03 \mathrm{D}$, at operating conditions typical of the present study. The air flow for the jet was routed through a small plenum chamber fitted with flow conditioning units. The velocity profiles at the nozzle exit, with no flow in the wind tunnel $\left(U_{T}=0\right)$, were found to be uniform (top hat). This is shown by the data in Fig. 2 measured with a Pitot probe for two flow rates. The average jet speed $\left(V_{j}\right)$, with or without the tunnel flow on, was determined from measurement of the mass flow rate via an orifice meter fitted to the supply line.

In view of the volume of the parameter space and the time consuming nature of the experiments, judgement had to be exercised in the choice of the flow parameters and tab configurations. Data were acquired for two values of the momentum-flux ratio, $J\left[=\left(\rho_{j} V_{j} / \rho_{T} U_{T}\right)^{2}\right]$, of 21.1 and 54.4 . The tunne! velocity for the two conditions was held at 6.77 and $4.18 \mathrm{~m} / \mathrm{s}$, respectively. Given the nozzle and tunnel geometry, the two values of $J$ represented extrema at which the jet trajectories were located suitably within the test section (not too close to the floor or the ceiling) permitting detailed 


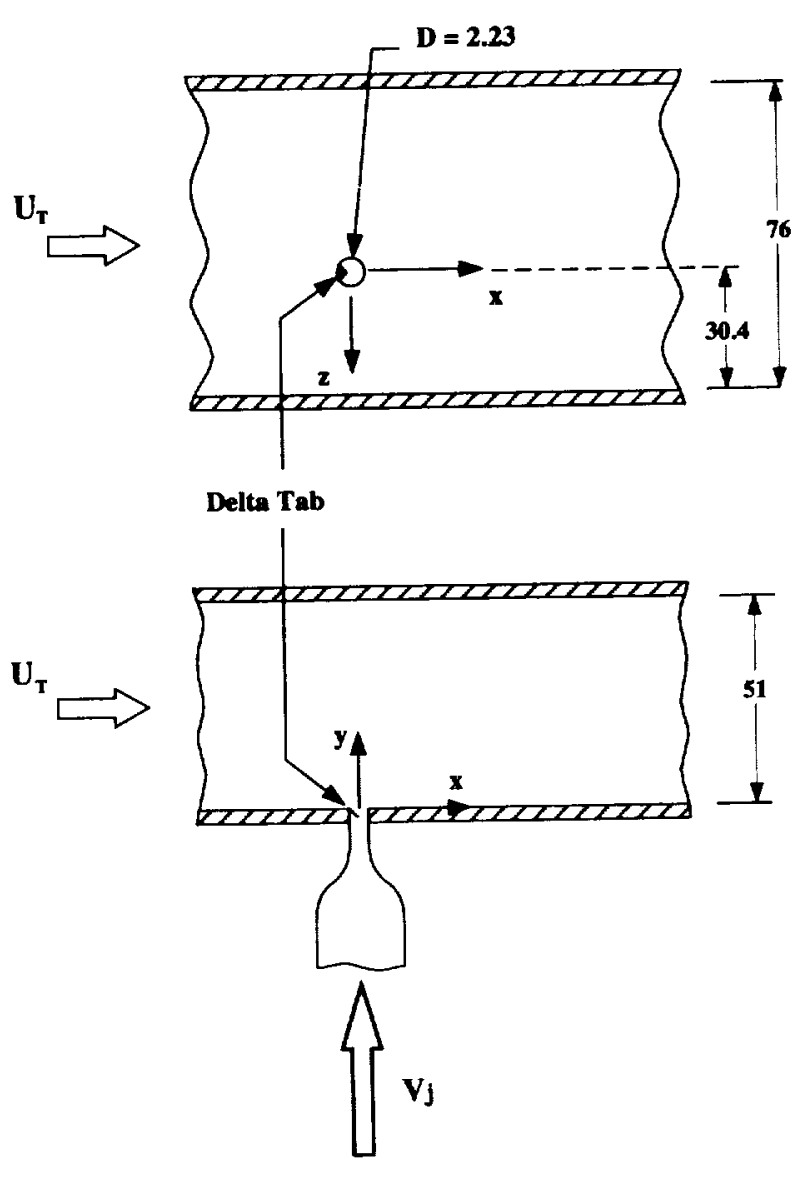

FIG. 1. Schematic of wind tunnel test section with cross-flow jet. Dimensions are in $\mathrm{cm}$.

flow field measurements. Note that a smaller jet diameter $(D)$ would allow these measurements at higher values of $J$, and vice versa. On the other hand, a relatively large $D$ was required from probe resolution considerations. These constraints led to the choice of the nozzle geometry for the given

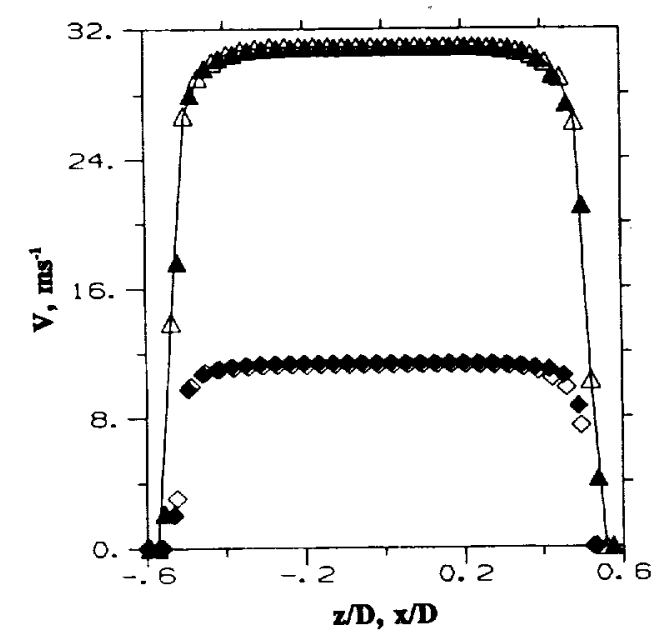

FIG. 2. Mean velocity profiles at jet nozzle exit for two flow rates with tunnel flow off $\left(U_{T}=0\right)$; solid symbols for $x$ profiles, open symbols for $z$ profiles (Fig. 1).

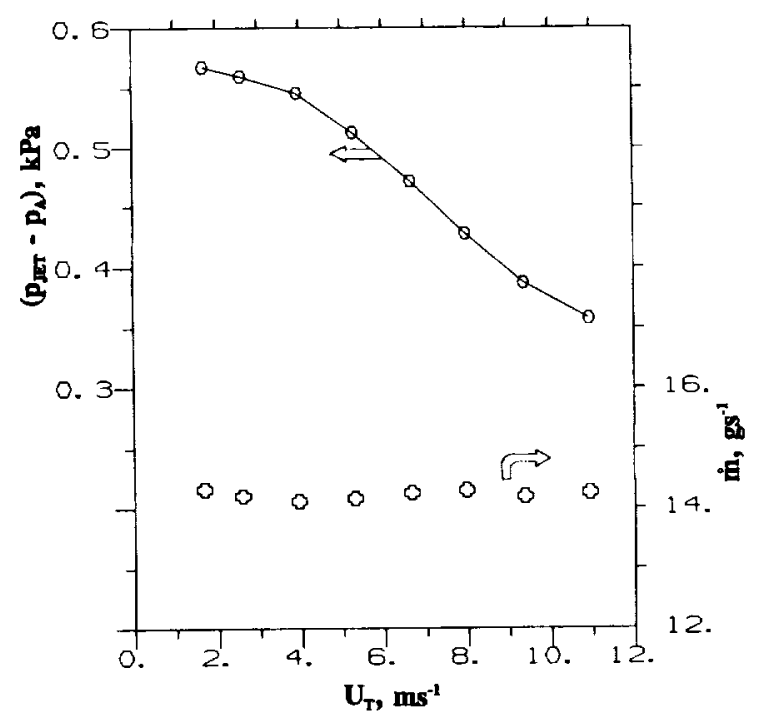

FIG. 3. For approximately constant flow rate $(\stackrel{m}{m})$, variation of jet plenum pressure $\left(p_{\text {jet }}\right)$ with tunnel speed $\left(U_{T}\right)$.

tunnel, which, in turn, yielded the aforementioned range of $J$. This range, however, covered or overlapped ranges investigated in most previous studies. ${ }^{5-10}$

The tab geometry was chosen based on limited parametric studies done previously with free jets. "Delta tabs" were used that had triangular shapes each with a base on the nozzle wall and an apex angle of $90^{\circ}$. The plane of the triangle made an angle of nominally $45^{\circ}$ with respect to the jet stream direction. It was found that for same area blockage this shape with a $45^{\circ}$ orientation (apex leaning downstream) produced the strongest effect in terms of mixing layer distortion. An explanation why this geometry was optimum for producing the strongest streamwise vortex pair was given in Ref. 2.

The tab size was chosen based on (unpublished) results of experiments in which the size was varied in a configuration involving four equally spaced delta tabs for a round free jet. It was found that the optimum increase in the jet entrainment with minimum thrust loss occurred for a size that corresponded to an area blockage of about $1.5 \%-2 \%$ per tab. Larger tab size yielded a minimal increase in entrainment with more thrust penalty. In the present study, a somewhat larger tab size ( $2.8 \%$ are a blockage) was chosen because thrust loss was not of primary concern; this was also the size used in Ref. 4 . The tab(s) with this shape and size was (were) placed at different locations on the periphery of the nozzle exit. In addition to the $+45^{\circ}$ orientation with respect to the jet stream direction, a $-45^{\circ}$ orientation was also tried for reasons explained in the next section. For ease of discussion, the tab configurations will be shown with small sketches with most of the datasets presented in the following. An open triangle will represent a tab with a $+45^{\circ}$ orientation, i.e., having its apex leaning up into the cross-flow, while a solid triangle will represent a tab with a $-45^{\circ}$ orientation, i.e., having its apex leaning inside the jet nozzle, as shown in Fig. 1.

Figure 3 shows, for the no-tab case, the variation of the 


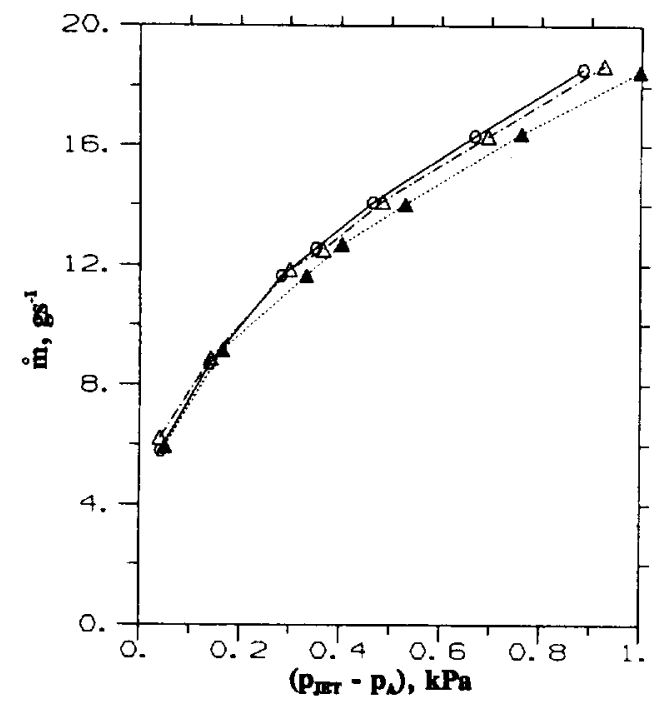

FIG. 4. For constant tunnel speed, $U_{T}=6.77 \mathrm{~ms}^{-1}$, variation of flow rate with plenum pressure for the jet: $O$, no tab; $\Delta$, one tab with apex leaning up by $45^{\circ} ; \boldsymbol{\Lambda}$, one tab with apex down by $45^{\circ}$ (Fig. 1).

jet plenum pressure $\left(p_{\text {jel }}\right)$ with tunnel speed while a constant flow rate for the jet is maintained; here $p_{A}$ is ambient pressure outside the tunnel. Since the tunnel is an induction-type one, subambient pressure exists within the test section when the tunnel flow is on. Thus, a drop in $p_{\text {jet }}$ is expected for the constant differential pressure required to maintain the constant flow rate. However, the drop in $p_{\text {jet }}$ is found to be much more than the expected drop in the tunnel pressure. For example, at $U_{T}=6.77 \mathrm{~ms}^{-1}, p_{\text {jet }}$ has dropped by $86 \mathrm{~Pa}$, whereas the tunnel pressure should be only $28 \mathrm{~Pa}$ below ambient. The observed pressure drop is further discussed in Sec. III.

While the area blockage due to each tab was $2.8 \%$ of the nozzle exit area, the actual fluid dynamic blockage was relatively more for the apex-down case. This is expected because the flow upstream of the tab in that case is brought to a halt, whereas for the apex-up case the flow is only slowed down. The higher blockage resulting in a lower flow rate for the former tab case can be seen in the data presented in Fig. 4. In the flow regime under consideration, the blockage was determined to be nominally $5.5 \%$ and $1.5 \%$ for the apex-down and apex-up cases, respectively. These yielded correction factors for the jet equivalent diameter of 0.972 and 0.992 , respectively. (Limited data showed that the blockage values did not change significantly when the tab location on the periphery of the nozzle exit was changed, or when $U_{T}$ was changed.) The appropriately corrected jet diameter was used for all data normalization.

Hot-wire measurements were carried out on a crosssectional $(y, z)$ plane of the jet at various streamwise $(x)$ locations. Two X-wire probes, one in the " $u-v$ "' and the other in the " $u-w$ " configuration, were traversed successively through the same grid points to obtain all three components of velocity as a function of $y$ and $z$. The finite separation of the sensors in the $X$ element $(0.040 \mathrm{in}$.) introduced errors in the $V$ and $W$ data due to $U$ gradients, which were corrected appropriately. ${ }^{2}$ The gradients of the corrected $V$ and $W$ provided streamwise vorticity $\omega_{x}$. Further details of the hot-wire measurement technique can be found in Refs. 2 and 14.

\section{RESULTS AND DISCUSSION}

Figure 5 shows the mean velocity and streamwise vorticity distributions measured at two $x / D$ locations for $J=21$ for the no-tab case. The mean velocity distributions reveal the characteristic "kidney shaped" structure. A rising column of low momentum fluid pushes into the band of higher momentum jet fluid rendering the latter into the characteristic kidney shape. Such a shape has been observed in many previous studies for velocity distributions, ${ }^{6-8}$ as well as for temperature distribution in nonisothermal flows, ${ }^{6}$ and concentration distributions in chemically reacting and nonreacting flows. 15,16

The $\omega_{X}$ data in Fig. 5 clearly identify the counterrotating "bound vortex pair." Initially, the vorticity is concentrated in two arcs spanning the shear layer regions on either side of the symmetry plane. Farther downstream, vorticity on each side migrates to form the rounded cores of the bound vortex pair. The solid and the dashed $\omega_{X}$ contours represent anticlockwise and clockwise rotations, respectively. Thus, the sense of the bound vortex pair is such that fluid from underneath is ingested and ejected upward. It may be recalled here, with reference to previous work on free jets, ${ }^{2}$ that the pair of streamwise vortices generated by a tab has a sense of rotation such that fluid from near the base of the tab is forced toward the apex, i.e., toward the core of the jet. Therefore, it should be apparent that the vortex pair generated by the tab, placed on the leeward side, would be of the same sense as that of the bound vortex pair. This led to the expectation, as stated in the Introduction, that the tab placed on the leeward side ought to strengthen the bound vortex pair. Conversely, a tab placed on the windward side would be expected to weaken the bound vortex pair.

It may also be observed that the peak values of vorticity in Fig. 5, when nondimensionalized as $\omega_{X} D / V_{j}$, are about 0.3 and 0.1 at $x / D=2$ and 8 , respectively. These vorticity magnitudes are comparable to those produced by a tab in a free jet. ${ }^{14}$ Thus, it is natural to expect that the tab would have a significant impact on the vorticity field of the flow under consideration, augmenting or cancelling the bound vortex pair when placed on the leeward or the windward side, respectively.

The effect of tab(s), placed in various configurations, on the mean velocity distribution is shown in Fig. 6. As mentioned before, the tab configuration in each case is shown by the inserted sketches; the arrow denotes the cross-flow direction. It is clear, in agreement with the observation of Ref. 4, that placing the tab on the leeward side [in (b)] has little effect compared to the no-tab case [in (a)]. Two tabs, $90^{\circ}$ apart, on the leeward side [in (c)] may have increased the jet penetration slightly and diffused the jet cross-section somewhat, but the effect is marginal. In comparison, significant effects are noticed in cases (d), (e), and (f). Specifically, when the tab is placed on the windward side, the apex leaning either up or down, the jet penetration is substantially reduced. In Ref. 4, a reduction in jet penetration was also noted when two tabs, one on the windward side and one on 

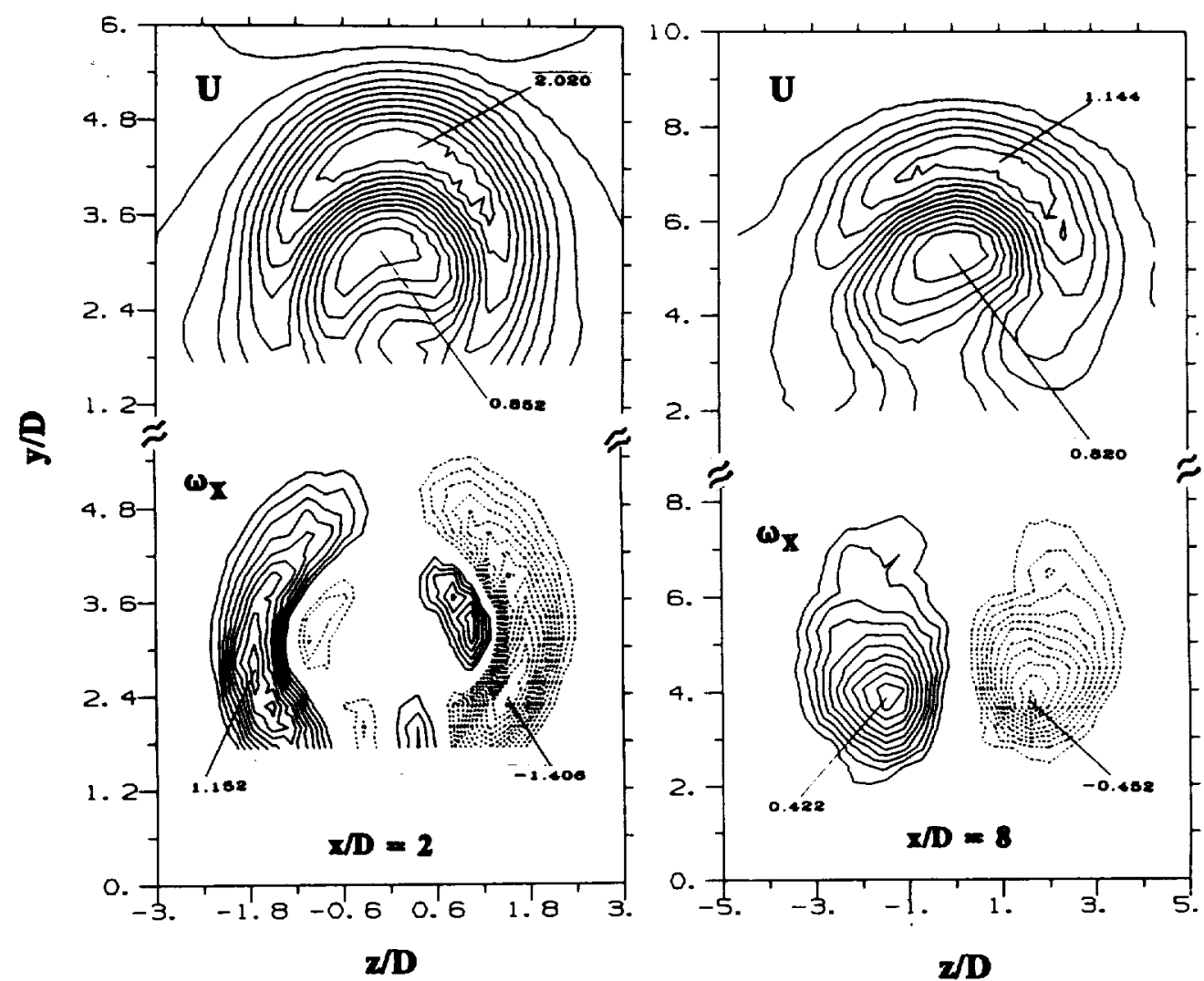

FIG. 5. For $J=21$, mean velocity $\left(U / U_{T}\right)$ and streamwise vorticity $\left(\omega_{X} D / U_{T}\right)$ distributions. Contour increments are 0.097 and 0.027 for velocity, and 0.107 and 0.037 for vorticity, at $x / D=2$ and 8 , respectively.

the leeward side, were used. Apparently, the effect of the tab on the windward side prevailed in that configuration.

In the case of Fig. 6(d), one finds that the velocity distribution has been drastically upset. This has occurred presumably due to a slight asymmetry in the placement of the tabs. Such a sensitivity is interesting, and although it remains far from clearly understood, it delineates the potential for flow control via appropriate manipulation of the nozzle exit. In this regard, it is noteworthy that visualization experiments reported by Wu et al. ${ }^{10}$ also demonstrated an acute sensitivity of the jet structure to nozzle shape, orientation, as well as to periodic perturbation.

Experiments at the higher $J(=54)$ yielded essentially similar results. As shown in Fig. 7, two tabs on the leeward side [in (b)] have little effect, while one tab on the windward side [in (c)] has reduced the jet penetration considerably.

Detailed distributions of velocity and vorticity were measured for the tab on the windward side case (apex down), as compared to the no-tab case. With reference to the discussion in Sec. II, although the apex-up case showed equal or greater effect [compare Figs. 6(e) and 6(f)] for less blockage, the apex-down case was chosen from certain practical considerations. The tab on the windward side would be exposed to hot flow in a combustor, and thus, the apex-down geometry was preferable from thermal and structural considerations. ${ }^{17}$ The data were acquired over a volume of the flow field. For brevity, only composite plots of these data are shown in Figs. 8 and 9 for $J=21$ and 54, respectively.
The plots provide a perspective view of the flow field evolution. The $U$ distributions show the outline of the jet, and it should be apparent that the effect of the tab at either $J$ is to bend the jet farther toward the tunnel floor, i.e., to cause less penetration. The streamwise vorticity data, shown by the two isosurfaces $\left(\omega_{X} D / U_{T}= \pm 0.16\right)$, depict the evolution of the bound vortex pair. The tab has clearly reduced the strength of the bound vortex pair, as evident from a reduction in the size of the vortices marked by the isosurfaces. The effect of the tab is similar for the two values of $J$.

From the data of Figs. 8 and 9, details of the jet evolution, with and without the tab, were further explored. From the mean velocity data, the penetration height of the jet was determined. The penetration height variation with streamwise distance, i.e., the jet trajectory, was investigated in most previous works dealing with the subject. ${ }^{6,10,11,15,16}$ Following Kamotani and Greber, ${ }^{6}$ the height $y$-max was defined as the location of the maximum velocity on the symmetry plane $(z=0)$. The variation of $y$-max with $x$ is shown in Fig. 10 for the two values of $J$. Data are compared with correlation equations based on past experiments. ${ }^{18}$ It is clear that the correlation equation, stated in the figure caption, is followed well by the jet without the tabs. Use of the tabs, however, results in a significant deviation. It is worth noting here that Wu et al. ${ }^{10}$ reported a significant reverse effect, i.e., higher penetration, when the jet was pulsated at certain frequencies.

From the vorticity data an estimate of the overall 

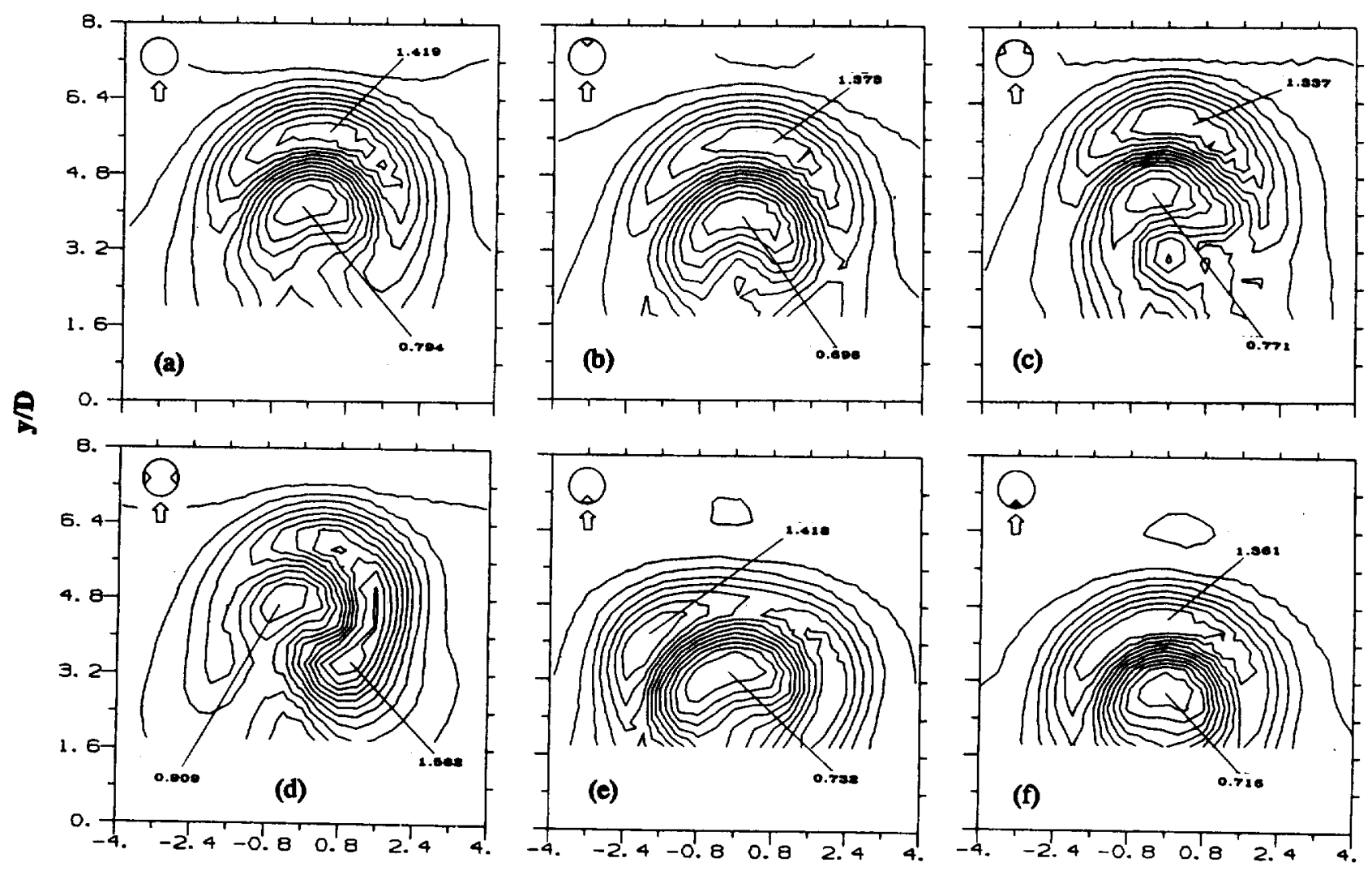

$\mathbf{z} / \mathbf{D}$

FIG. 6. For $J=21$, the effect of various tab configurations on the mean velocity ( $U / U_{T}$ ) distribution, at $x / D=4$. Contour increments (a) 0.052 , (b) 0.056 , (c) 0.047 , (d) 0.055 , (e) 0.057 , and (f) 0.054 .

strength of each component of the bound vortex pair was made. The circulation $\left(\Gamma=\iint \omega_{X} \partial y \partial z\right)$ was calculated for either side of the symmetry $(z=0)$ plane. (In most cases, the amplitudes of the circulation for the two sides of the symmetry plane agreed closely. The value of $\Gamma$ also checked well with that from line integration of $V$ and $W$.) The variation of $\Gamma$ with streamwise distance for the cases of Figs. 8 and 9 is shown in Fig. 11. Data at the upstream locations for some of the cases had to be omitted because segments of the vortices near the tunnel floor were missed by the measurement plane (see, for example, the $\omega_{X}$ data at $x / D=2$ in Fig. 5).

In the immediate vicinity of the nozzle, one might expect that the value of $\Gamma$ would approach zero because the $x$ component of vorticity associated with the bound vortex should be zero initially. Thus, the magnitude of $\Gamma$, for all the cases in Fig. 11, has gone through a sharp increase shortly down-
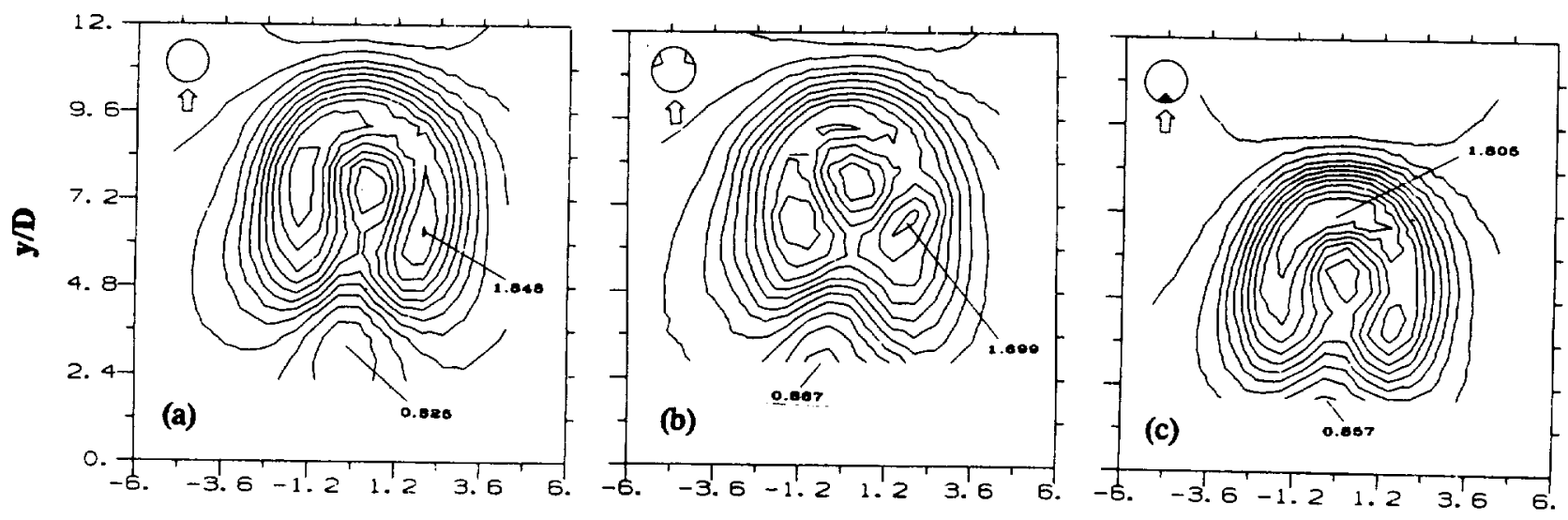

$\mathbf{2} / \mathbf{D}$

FIG. 7. For $J=54$, the effect of various tab configurations on the mean velocity distribution, at $x / D=4$. Contour increments (a) 0.085 , (b) 0.069 , and (c) 0.079 . 

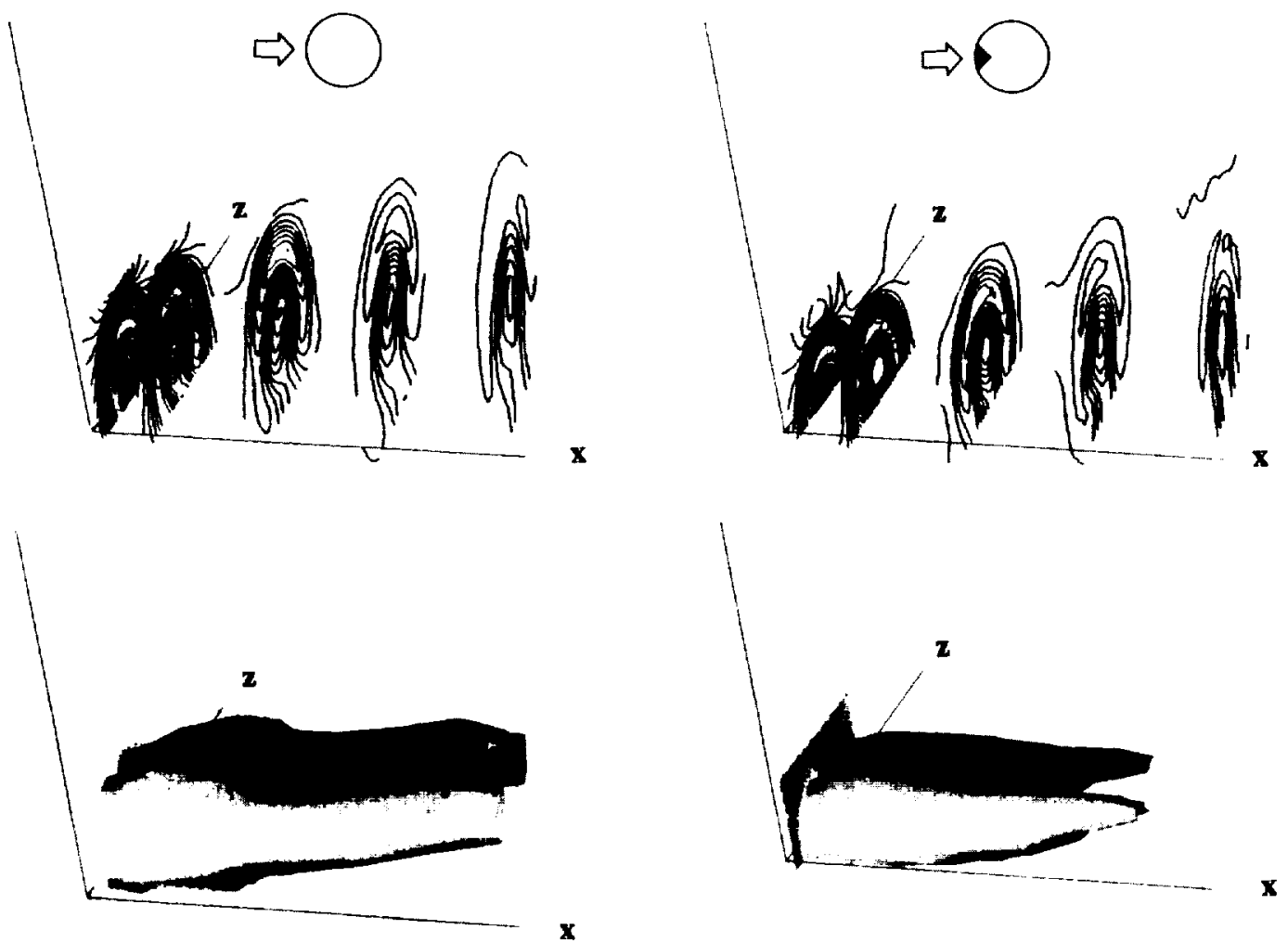

$\mathbf{x}$

FIG. 8. For $J=21$, the perspective views of mean velocity (top) and streamwise vorticity (bottom) distributions, based on data at $x / D=2,4.8,12$, and 16 . Left column: without a tab, right column: with a tab.

stream of the nozzle while the jet has been bent by the crossstream. Thereafter, a gradual decrease in the value of $\Gamma$ takes place with increasing $x$ apparently due to the action of turbulent diffusion. Most remarkable is the fact that the tab, at either value of $J$, has substantially reduced the strength of the bound vortices.

The bound vortex strength for the tab cases of Fig. 6 is now examined in a similar manner. The values of $\Gamma$, from each half of the symmetry plane, for the six cases of Fig. 6 are listed in Table I. A significant reduction in the bound vortex strength is again obvious when the tab is placed on the windward side. [As discussed earlier, the lower values in (e) and (f) are partly due to the fact that small segments of the vortices near the tunnel floor were missed by the measurement range.] Note that in case (d), the negative circulation dominates, implying a significant net clockwise circulation over the entire jet cross-section. This is consistent with the clockwise tilting of the velocity distribution seen in Fig. 6(d). Presumably, this has occurred due to minor nonsymmetry in the placement of the two tabs, again, demonstrating the sensitivity of the vortex system to slight perturbations at the jet exit.

Note also from Table I that in cases (b) and (c) the tabs have not changed the circulation compared to the no-tab case value in (a). This is consistent with the almost unchanged mean velocity distributions seen in Figs. 6(b) and 6(c). Why is the tab ineffective when placed on the leeward side? An answer to this question traces to the static pressure distribution in the vicinity of the nozzle exit.

Static pressure distributions, measured on the leeward side of the nozzle for the no-tab case at $J=21$, are shown in Fig. 12. The data, obtained by a probe traversed about $1 \mathrm{~mm}$ from the tunnel floor, approximate the wall static pressure $\left(p_{\mathrm{ST}}\right)$ distribution. A steep drop in the static pressure near the twelve o'clock position on the leeward side is obvious. Note that away from the nozzle the pressure coefficient, $C_{p}\left[=\left(p_{\mathrm{ST}}-p_{A}\right) 1 / 2 \rho U_{T}^{2}\right.$, where $p_{A}$, again, is ambient pressure outside the tunnel], relaxes to a value somewhat lower than -1 due to losses in the flow conditioning units of the tunnel. Recall from Fig. 3 that the jet plenum pressure required to sustain a constant $V_{j}$ was found to drop substantially when the tunnel flow was turned on. Scrutiny shows that the amount of that drop approximately corresponds to the static pressure drop occurring on the leeward side. Note also from the $z$ profiles in Fig. 12 that there occurs, relative to the tunnel pressure, a smaller positive $C p$ peak on the windward side of the jet.

The wall static pressure distribution for similar flows has been measured and reported in previous studies. ${ }^{18,19}$ The $C p$ contours presented in these references were for higher values of $J$ but corroborate the trend observed in Fig. 12. With the aid of the data from the cited references the expected $C p$ distribution around the periphery of the present nozzle is constructed schematically in Fig. 13. On the windward side (six o'clock position) a narrow lobe of slightly positive $C p$ occurs (relative to the test section pressure). At about seven and five o'clock positions the $C p$ values go through a steep drop. Subambient pressures occur around the rest of the nozzle with $C p$ being about -3 at the twelve o'clock position (Fig. 12). Even lower $C p$ values might occur on the 

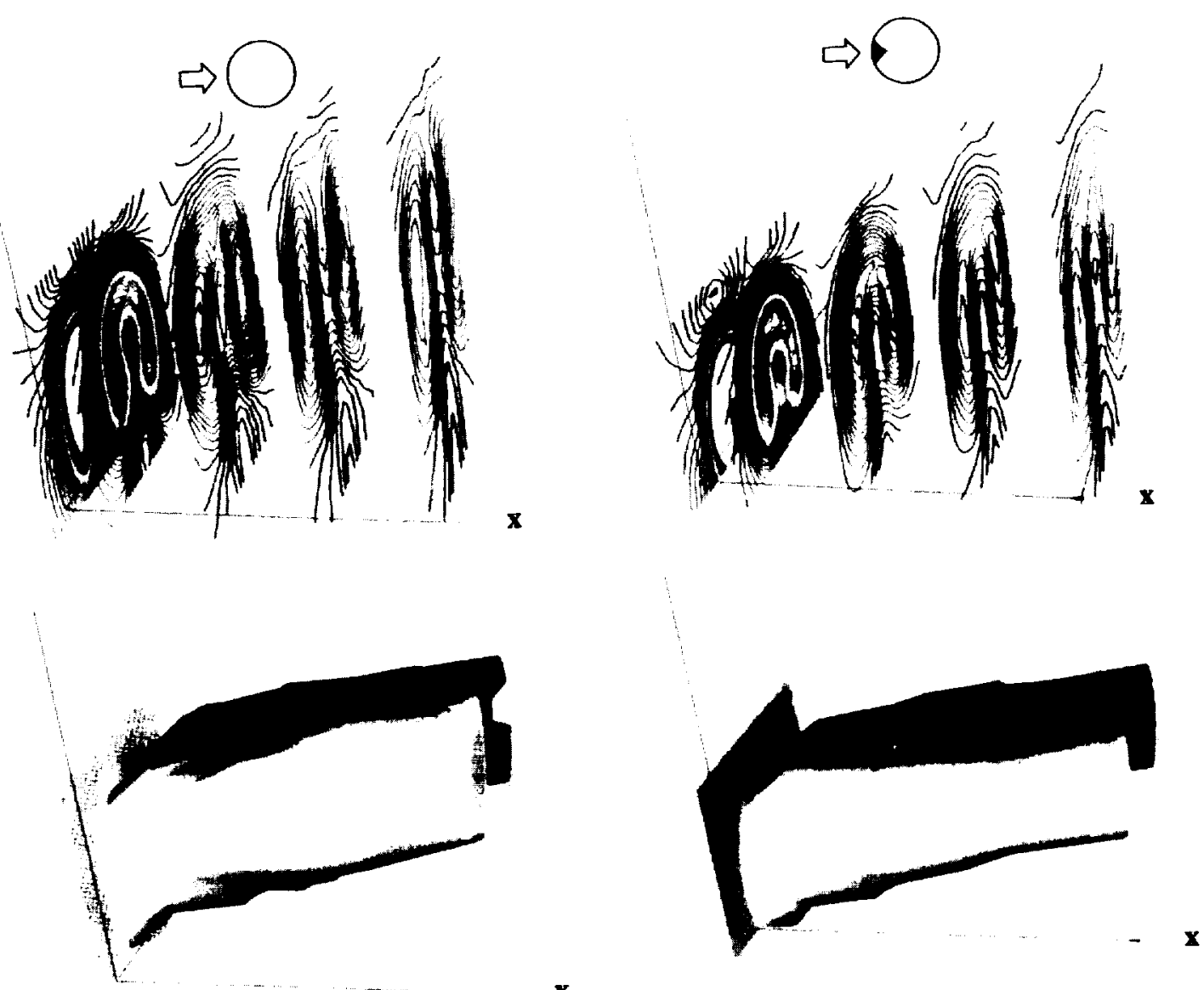

FIG. 9. For $J=54$, the perspective views of data as in Fig. 8 .

sides where the two bound vortices are apparently anchored. ${ }^{19}$ The low pressures all around the nozzle, except for a narrow segment on the windward side, occur presumably because of the streamline curvature associated with the jet itself and the cross-stream flow going around it.

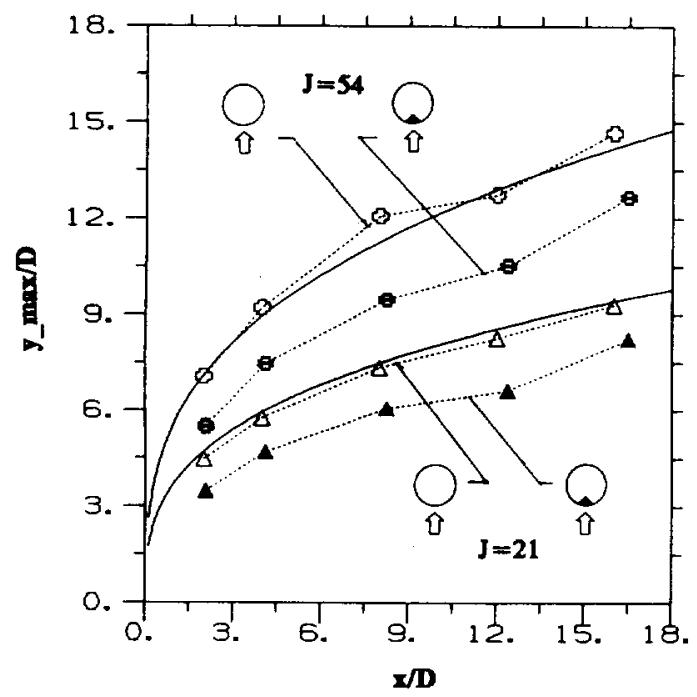

FIG. 10. The height of the maximum mean velocity location (at $z=0$ ) as a function of $x / D$. Solid lines represent the equation, $y=J^{0.433} * x^{0.333}$ for the two values of $J$ (Ref. 18)
It is the low static pressure that is thought to cause the ineffectiveness of the tab when placed on the leeward side. The main source of streamwise vorticity from a tab is a "pressure hill" generated just upstream of the tab. The lateral pressure gradients of the hill together with the presence

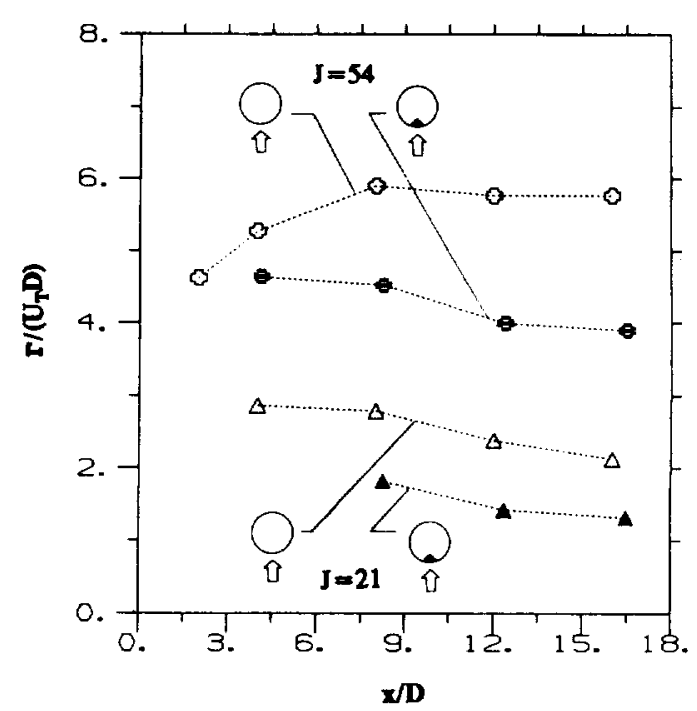

FIG. 11. Circulation on one side of the symmetry $(z=0)$ plane with and without a tab for the two values of $J$. 


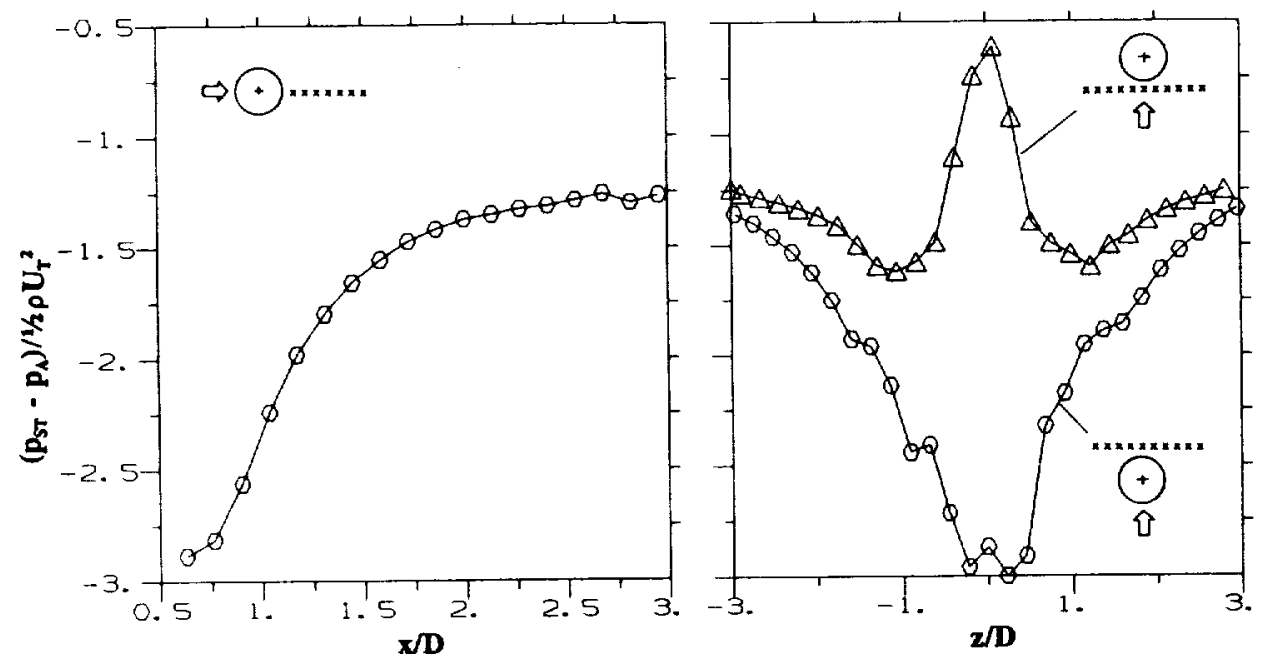

FIG. 12. Static pressure distribution in the vicinity of the jet exit.

of the nozzle wall become the source for the pair of counterrotating vortices. ${ }^{2}$ For the present flow, the already existing lower pressure on the leeward side negates the formation of a pressure hill with large amplitude. In a simplistic sense, referring back to the $p(z)$ distribution in Fig. 12 for the leeward side, it can be seen that the tab has to generate a pressure hill where there already exists a pressure valley. Thus, a resultant pressure hill with diminished amplitude renders the tab less effective. A comparable example is an overexpanded supersonic jet flow. ${ }^{2}$ Depending on the extent of overexpansion, even an adverse pressure gradient may exist near the nozzle wall at the exit plane. The tab in that case does not produce a pressure hill and becomes completely ineffective. It should be noted that the complete ineffectiveness of the tab in the present case, when placed on the leeward side, is somewhat surprising. An inspection of the pressure data at the given $J$ makes it apparent that the tab placed at that location should still produce a pressure hill of considerable amplitude, and thus, there should be some effect. The virtual ineffectiveness is probably due to subtle complexities in the flow at the nozzle exit, which are not yet fully understood.

In contrast to the effect on the leeward side, the positive static pressure on the windward side augments the pressure hill and explains the effectiveness of the tab when placed there. The tab in this case, as discussed earlier, however, generates vorticity with a sense counter to that of the bound vortex pair. The amplitude of the latter pair is thus diminished and the jet penetration reduced. Note that such an ef-

TABLE 1. Circulation, $I / U_{1} D$, on the left and right halves of the $z=0$ plane for the tab cases of Figs. 6(a)-6(f).

\begin{tabular}{ccc}
\hline \hline Case & Left & Right \\
\hline (a) & 2.860 & -2.824 \\
(b) & 2.877 & -2.912 \\
(c) & 2.855 & -2.864 \\
(d) & 2.905 & -3.260 \\
(e) & 2.327 & -2.195 \\
(f) & 1.856 & -1.735 \\
\hline
\end{tabular}

fect may in fact reduce mixing of the jet with the cross-flow, and the impact on mixing for a given jet penetration with and without the tabs remains to be explored. The reasoning provided in the foregoing, however, qualitatively explains the primary effects.

\section{CONCLUSION}

The present results confirm the ineffectiveness of the tab when placed on the leeward side of the nozzle issuing the jet into the cross-flow, as reported by Liscinsky et al. ${ }^{4}$ A study of the static pressure distribution provides an explanation. The region in the vicinity of the leeward side of the nozzle is characterized by low static pressures. Thus, when placed on that side, the tab generates a "pressure hill" with a severely diminished amplitude. Since the pressure hill is the primary source of streamwise vorticity in the flow over a tab, the diminished amplitude qualitatively explains the ineffectiveness of the tab. Comparatively, a significant effect is observed when the tab is placed on the windward side. As expected, the tab in this configuration weakens the bound vortex pair. This in turn reduces the penetration of the jet.

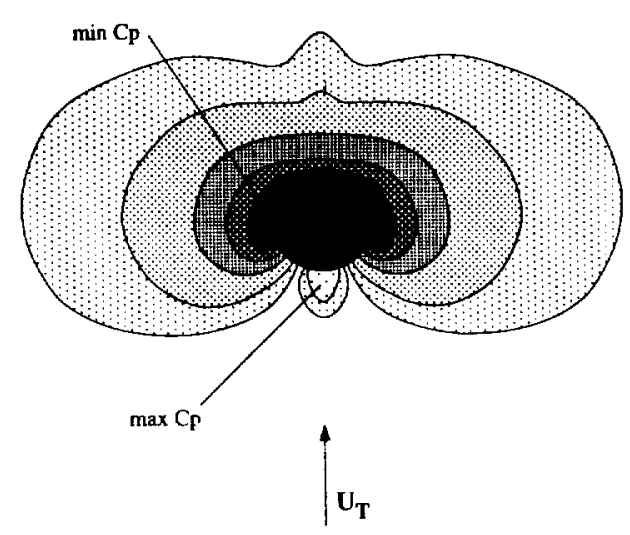

FIG. 13. Schematic of the expected pressure distribution around the nozzle, after Refs. 20 and 21. 
'K. K. Ahuja and W. H. Brown, "Shear flow control by mechanical tabs," AIAA Paper No. 89-0994, 1989.

${ }^{2}$ K. B. M. Q. Zaman, M. F. Reeder, and M. Samimy, "Control of an axisymmetric jet using vortex generators," Phys. Fluids A 6, 778 (1993)

${ }^{3}$ K. B. M. Q. Zaman, "Spreading characteristics and thrust of jets from asymmetric nozzles," AIAA Paper 96-0200, 34th Aerospace Sciences Meeting, Reno, Nevada, 15-18 January 1996.

${ }^{4}$ D. S. Liscinsky, B. True, and J. D. Holdeman, "Effects of initial conditions on a single jet in crossflow," AIAA Paper 95-2998, 31 st Joint Propulsion Conference. San Diego, 10-12 July 1995.

5 J. F. Keffer and W. D. Baines, "The round turbulent jet in a cross-wind," J. Fluid Mech. 15, 481 (1963).

${ }^{6} \mathrm{Y}$. Kamotani and I. Greber, "Experiments on a turbulent jet in a cross flow," AIAA J. 10, 1425 (1972).

${ }^{7}$ R. Fearn and R. P. Weston, "Vorticity associated with a jet in a cross flow," AIAA J. 12, 1666 (1974).

${ }^{8}$ Z. M. Moussa, J. W. Trischka, and S. Eskinazi, "The near field in the mixing of a round jet with a cross-stream," J. Fluid Mech. 80, 49 (1977).

"T. F. Fric and A. Roshko, "Vortical structure in the wake of a transverse jet," J. Fluid Mech. 279, 1 (1994).

${ }^{10} \mathrm{~J} . \mathrm{M}$. Wu, A. D. Vakili, and F. M. Yu, "Investigation of the interacting flow of nonsymmetric jets in crossflow," AIAA J. 26, 940 (1988).

${ }^{1 " A}$. Lozano, S. H. Smith, M. G. Mungal, and R. K. Hanson, "Concentra- tion measurements in a transverse jet by planar laser-induced fluorescence of acetone," AIAA J. 32, 218 (1994).

${ }^{12} \mathrm{P}$. Huq and M. R. Dhanak, "The bifurcation of circular jets in crossflow," Phys. Fluids 8, 754 (1996).

${ }^{13}$ B. D. Pratte and M. Baines, "Profiles of the round turbulent jet in a crossflow," J. Hydronaut. Div. ASCE 92, 53 (1967).

${ }^{14} \mathrm{~K}$. B. M. Q. Zaman, "Axis switching and spreading of an asymmetric jet-The role of coherent structure dynamics," J. Fluid Mech. 316, 1 (1996).

${ }^{15}$ J. E. Broadwell and R. E. Breidenthal, "Structure and mixing of a transverse jet in incompressible flow," J. Fluid Mech. 148, 405 (1984).

${ }^{16}$ D. S. Liscinsky, B. True, and J. D. Holdeman, "Crossflow mixing of noncircular jets," J. Prop. Power 12, 225 (1996).

${ }^{17}$ J. D. Holdeman (private communication, 1995).

${ }^{18} \mathrm{G}$. N. Abramovich, "The theory of turbulent jets," The M.I.T. Press, 1963.

${ }^{19}$ J. F. Foss (private communication, 1996).

${ }^{20}$ L. J. S. Bradbury and M. N. Wood, "The static pressure distribution around a circular jet exhausting normally from a plane wall into an airstream," A.R.C. CP 822, 1965.

${ }^{21} \mathrm{H}$. M. McMahon and D. K. Mosher, "Experimental investigation of pressures induced on a flat plate by a jet issuing into a subsonic cross wind," NASA SP 218, 1969. 


